\title{
Scénarisation des mesures dans l'environnement pour un exercice national de crise : l'exemple de l'exercice de Saclay du 17 septembre 2009
}

\author{
N. COMTE ${ }^{1}$, L. BOURGOIS ${ }^{1}$, M. MAZIÈRE ${ }^{2}$
}

(Manuscrit reçu le 25 janvier 2010, accepté le 7 mars 2010)

RÉSUMÉ Le 17 septembre 2009, un exercice de crise national a eu lieu sur le site du Commissariat à l'énergie atomique (CEA) de Saclay. Le scénario choisi était la fusion à l'air d'un élément combustible du réacteur de recherche OSIRIS. L'objectif de cet exercice était d'atteindre le niveau d'intervention conduisant à la distribution d'iode stable à Villiers-le-Bâcle. Pour que l'exercice puisse se dérouler de façon optimale, il était important de simuler un ensemble d'appareils utilisés par les personnels réalisant les mesures pour le compte des pouvoirs publics ou de l'exploitant et ceci à n'importe quel endroit de l'espace et moment de la mesure. Au vu du grand nombre de données à fournir en temps réel, il était illusoire de vouloir utiliser un logiciel de calcul de type dispersion atmosphérique le jour de l'exercice. En effet, les temps de réponse de ce type de code ne sont pas compatibles avec la réponse opérationnelle attendue par les acteurs de l'exercice. Il a donc été décidé de mettre au point une méthode permettant de répondre rapidement à chacune des demandes des acteurs de terrain, quelle que soit la grandeur demandée, l'heure de la demande et la position du point de mesure. Cet article décrit la méthode et les outils ayant permis de simuler ces grandeurs. Douze grandeurs ont été simulées en 1000 points de mesures dans un domaine spatial de $60 \mathrm{~km}^{2}$ et pour 18 instants de calcul, le tout représentant plus de 200000 valeurs. La préparation a demandé 6 semaines de travail. Ceci a permis aux scénaristes de répondre à l'ensemble des sollicitations des acteurs de terrain dans des temps très rapides. Les personnels assurant les mesures ont réalisé des mesures sur environ 250 points (dans l'espace et le temps) représentant de l'ordre de 1000 valeurs.

ABSTRACT Environmental assessment for a radiological emergency management exercise The example of the Saclay exercise, on September 17, 2009.

On September 17, 2009, a radiological emergency management exercise took place on the site of Saclay site of the French Atomic Energy Commission. The selected scenario was the in-air melting of a fuel element from the nuclear research reactor "OSIRIS". The aim of the exercise was to reach a level of releases leading to the distribution of stable iodine in Villiers-le-Bâcle. In order to optimize the exercise, it was important to simulate all the measuring devices used by the actors, at any place and any time during the crisis. Considering the large amount of data to provide in real time, it was unrealistic to use computation software, such as atmospheric dispersion code, the day of the exercise. Current software response delay is not

CEA, DAM, DIF, 91297 Arpajon, France.

2 CEA, DSM/SAC/DIR, 91191 Gif-sur-Yvette Cedex, France. 
compatible with the operational aspect of an exercise. It was therefore decided to develop a method to quickly answer each actor's request, whatever the measurement, time or location. This article describes the method and the tools, referencing the scenario, which allowed the simulation of these quantities. Twelve measurements were simulated at 1000 points, in a spatial area of $60 \mathrm{~km}^{2}$ and for 18 calculation times. All of the data represents more than 200000 values, and the preparation took 6 weeks. This made it possible for the scenario writers to respond to all actors' requests in a very short time. The participants used approximately 250 simulated measurements (location and time), representing about 1000 values.

Keywords: Accident in reactor / environmental impact / environmental monitoring / reactor (research)

\section{Introduction}

Le 17 septembre 2009, un exercice national de crise a eu lieu sur le site du Commissariat à l'énergie atomique (CEA) de Saclay. L'installation choisie était le réacteur de recherche OSIRIS. La définition du scénario de l'exercice ainsi que l'ensemble des mesures radiologiques ont été confiés au CEA. La partie concernant la simulation des mesures dans l'environnement a été confiée au Service de protection contre les rayonnements du CEA/DAM Ile-de-France. Pour que l'exercice se déroule de façon optimale et présente un intérêt pour les intervenants sur le terrain, la réponse d'un ensemble d'appareils de mesure pouvant être utilisés par les acteurs de terrain a dû être simulé.

Les grandeurs de radioprotection mesurables dans l'environnement sont les suivantes :

- le débit d'équivalent de dose,

- l'activité surfacique (pouvant être associée à un spectre),

- l'activité volumique dans l'air (pouvant être associée à un spectre) ou son intégrale.

La valeur de chacune de ces grandeurs dépend du moment (à partir du début de l'accident, nommé $T_{0}$ par la suite) et de l'endroit de la mesure. Les appareils de mesure ont une réponse spécifique en fonction du spectre et/ou de la forme physico-chimique de l'élément : par exemple, la réponse des appareils mesurant l'activité surfacique est donnée en coups par seconde (noté c/s dans la suite du document), les filtres des appareils de prélèvement atmosphérique piègent soit des aérosols soit des halogènes...

Certains instruments mesurent la grandeur de manière continue, fait dont il a fallu tenir compte dans l'élaboration des méthodes de calcul.

Ainsi, au vu du grand nombre de données à fournir en temps réel aux personnels réalisant les mesures pour le compte des pouvoirs publics ou de 
l'exploitant (nombre de grandeurs, de points dans l'espace, des instants de calcul), dans un scénario complexe (cinétique de rejet, nombre de radionucléides), il était illusoire de vouloir utiliser un logiciel de calcul de type dispersion atmosphérique le jour de l'exercice. En effet, les temps de réponse de ce type de code ne sont pas compatibles avec la réponse opérationnelle attendue par les acteurs de l'exercice. Il a donc été décidé de mettre au point une méthode permettant de répondre rapidement à chacune des demandes des acteurs de terrain dans un format facilement exploitable, ceci quelle que soit la grandeur demandée, l'heure de la demande et la position du point de mesure dans l'espace.

\section{Matériel et méthode}

Chacune des grandeurs calculées dans l'environnement peut être reliée au temps par l'intermédiaire de l'activité rejetée à la cheminée et à sa position dans l'espace par le coefficient de transfert atmosphérique (CTA). Ce coefficient est fonction de la position du point de calcul dans l'espace, de la hauteur de rejet et des conditions météorologiques. Si les conditions météorologiques ne varient pas (ce qui était le cas lors pour l'exercice), le CTA ne dépend plus du moment d'observation mais uniquement de sa position dans l'espace.

De même, chacune des grandeurs peut être reliée à son instant de mesure $(t)$ par l'activité ou le débit d'activité à la cheminée au même instant $t$. Cette formulation n'est valable que dans l'hypothèse, acceptable au vu des distances considérées, que le temps de transfert entre le point de rejet et le point de mesure est négligeable.

Ces relations sont caractérisées par les formules classiques données par les équations (1) à (5) :

$$
\begin{gathered}
a_{v}(x, y, t)=a_{c h}(t) \times C T A(x, y) \\
A_{s}(x, y, t)=A_{c h}(t) \times v_{d} \times C T A(x, y) \\
\dot{H}(x, y, t)_{p a n}=C T A(x, y) \times \sum_{i} a_{c h, i}(t) \times F C D_{p a n, i} \\
\dot{H}(x, y, t)_{\text {depo }}=C T A(x, y) \times v_{d} \times \sum_{i} A_{c h, i}(t) \times F C D_{\text {depo }, i} \\
A_{v}(x, y, T)=\left(A_{c h}(T)-A_{c h}\left(T_{0}\right)\right) \times C T A(x, y)
\end{gathered}
$$

avec

$a_{v}(x, y, t):$ activité volumique mesurée au point $x, y$ et au temps $t$, 
$a_{c h}(t)$ : débit d'activité à la cheminée au temps $t$,

CTA $(x, y)$ : coefficient de transfert atmosphérique au point $x, y$,

$A_{s}(x, y, t)$ : activité surfacique mesurée au point $x, y$ et au temps $t$,

$A_{c h}(t)$ : activité intégrée à la cheminée au temps $t$,

$v_{d}$ : vitesse apparente de dépôt (égale à 0 pour les gaz rares),

$\dot{H}(x, y, t)_{\text {pan }}$ : débit d'équivalent de dose dû au panache au point $x, y$ et au temps $t$,

$a_{c h, i}(t)$ : débit d'activité à la cheminée au temps $t$ pour le radionucléide $i$,

$F C D_{\text {pan, } i}$ : facteur de conversion de dose panache pour le radionucléide $i$ (Kocher, 1983),

$\dot{H}(x, y, t)_{\text {depo }}$ : débit d'équivalent de dose dû au dépôt au point $x, y$ et au temps $t$,

$A_{c h, i}(t)$ : activité intégrée à la cheminée au temps $t$ pour le radionucléide $i$,

$F C D_{\text {depo, } i}$ : facteur de conversion de dose dépôt pour le radionucléide $i$ (Kocher, 1983),

$A_{v}(x, y, T)$ : activité volumique intégrée de $T_{0}$ à $T$.

En fonction de leur type, les appareils quantifiant l'activité volumique mesurent soit des aérosols, soit des halogènes soit des gaz. Aussi, pour simuler la réponse de l'ensemble des appareils de mesure d'activité volumique, il faut pouvoir discriminer l'activité volumique des gaz rares, des halogènes et des aérosols. Pour cela il suffit dans l'équation (1) (ou (5)) d'utiliser le débit d'activité (ou l'activité intégrée) des gaz, des halogènes ou des aérosols à la cheminée.

\section{Résultats : application au réacteur de recherche OSIRIS CEA/Saclay}

\subsection{Données de l'installation}

Pour l'exercice national du 17 septembre 2009, l'installation choisie était le réacteur de recherche OSIRIS. L'accident de référence concernait une fusion à l'air d'un élément combustible. Parmi les contraintes du scénario, il fallait déterminer un événement permettant de provoquer des rejets qui conduisent les autorités à décider de lancer la distribution de comprimés d'iode stable deux 


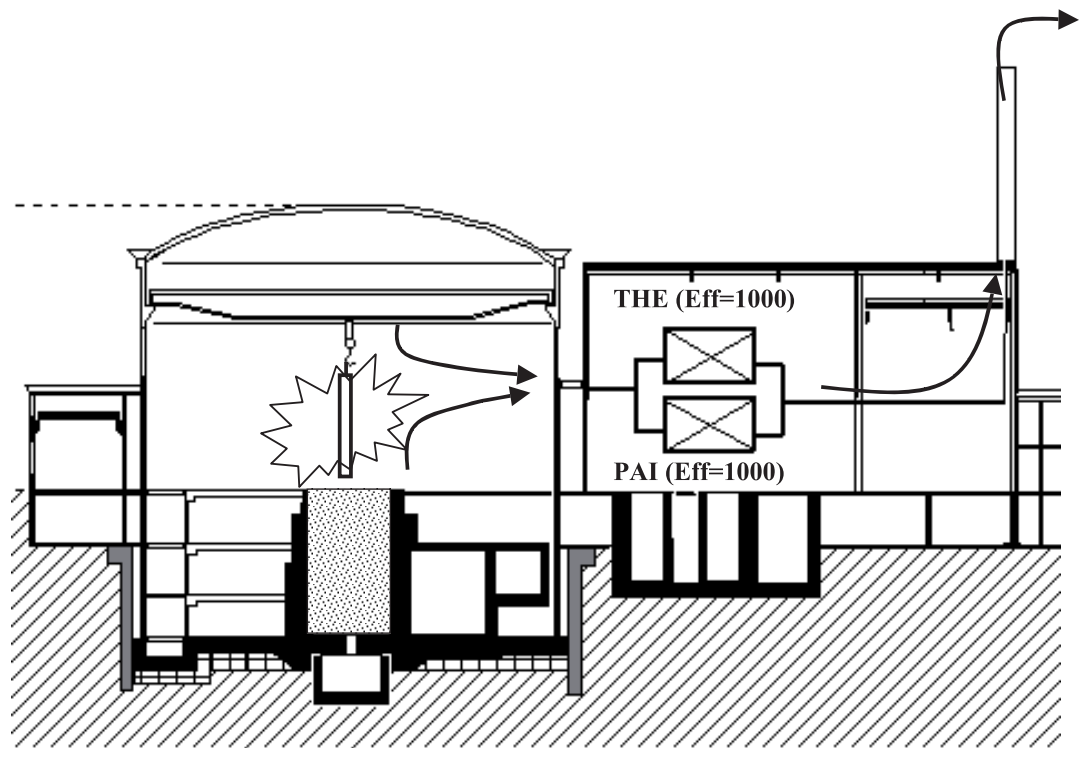

Figure 1 - Description schématique d'une fusion à l'air sur le réacteur OSIRIS.

Illustration of an in-air melting of a fuel element from OSIRIS reactor.

heures après le début de l'exercice à deux kilomètres du point d'émission. Pour cela, une dégradation de l'efficacité des filtres pièges à iode a été scénarisée à partir de $T_{0}+1 \mathrm{~h}$. Les pièges à iode avaient une efficacité nominale de 1000 de $T_{0}$ à $T_{0}+1 \mathrm{~h}$ puis voyaient leur efficacité divisée d'un facteur 10 toutes les trente minutes impliquant une perte totale d'efficacité à $T_{0}+2$ heures.

Une description schématique de l'installation est donnée sur la figure 1. Lors d'une fusion à l'air, un transfert instantané s'opère entre l'élément combustible et le hall du réacteur, le taux de transfert dépend de la forme physico-chimique de l'élément considéré. La pollution radioactive se répand alors dans le hall et est évacuée par le système de ventilation. Le polluant migre vers la cheminée en étant filtré par les filtres très haute efficacité (THE) pour les aérosols ou les pièges à iodes (PAI) pour les iodes.

\subsection{Codes de calcul}

Les grandeurs mesurables dépendent du débit d'activité et de l'activité intégrée à la cheminée. Ces grandeurs sont facilement accessibles pour des rejets ponctuels uniques de radionucléides de période relativement longue. Par contre, dans le cas d'un réacteur de recherche tel qu'OSIRIS, la cinétique de rejet, les périodes 
radioactives et les filiations de certains radionucléides considérés font que ces deux grandeurs évoluent rapidement dans le temps. Ainsi, pour leur évaluation, un code de calcul à compartiment a été utilisé : Erastem (Bourion et al., 1996 ; Comte et al., 2004, Monfort et al., 2008), basé sur l'algorithme de Birchall (Birchall, 1986 ; Birchall et James, 1989). Dans ce code, les transferts (fonction de la forme chimique des éléments) entre le combustible et le hall réacteur, la ventilation du hall réacteur, les taux de piégeages dans les filtres (très haute efficacité ou piège à iode) ainsi que les décroissances/filiations des radionucléides sont pris en compte. Le logiciel permet de faire varier dans le temps ces paramètres de transfert.

Les données d'entrée du code (transferts, ventilation, terme source combustible constitué d'une centaine de radionucléides) sont celles couramment utilisées pour une fusion à l' air dans le réacteur OSIRIS, à l'exception de la dégradation des filtres pièges à iode dont la variation de l'efficacité avec le temps a été simulée : nominale (efficacité de 1000) à $T_{0}$ puis décroissant d'un facteur 10 toutes les 30 minutes à partir de $T_{0}+1$ heure provoquant la perte totale d'efficacité à $T_{0}+2$ heures.

Les calculs ont été effectués de $T_{0}$ à $T_{0}+6$ heures (durée de l'exercice); par pas de 15 minutes jusqu'à $T_{0}+3$ heures puis par pas de 30 minutes jusqu'à $T_{0}+6$ heures.

\subsection{Activités et débit d'activité à la cheminée}

Les activités calculées par ERASTEM, $A_{c h}(t)$, pour les aérosols, les iodes et les gaz sont présentées sur la figure 2.

Le code ERASTEM permet également de calculer l'activité à la cheminée par radionucléides.

Les débits d'activités calculées par ERASTEM, $a_{c h}(\mathrm{t})$, pour les aérosols, les iodes et les gaz sont présentés sur la figure 3.

Cette figure permet de visualiser l'augmentation de l'activité des iodes consécutive à la dégradation des filtres PAI.

\subsection{Coefficient de transfert atmosphérique (CTA)}

Pour calculer les grandeurs en chaque point de l'espace, il faut disposer du coefficient de transfert atmosphérique (CTA). Ce coefficient est fonction de la position du point de mesure dans l'espace, de la hauteur de rejet et des conditions météorologiques. 


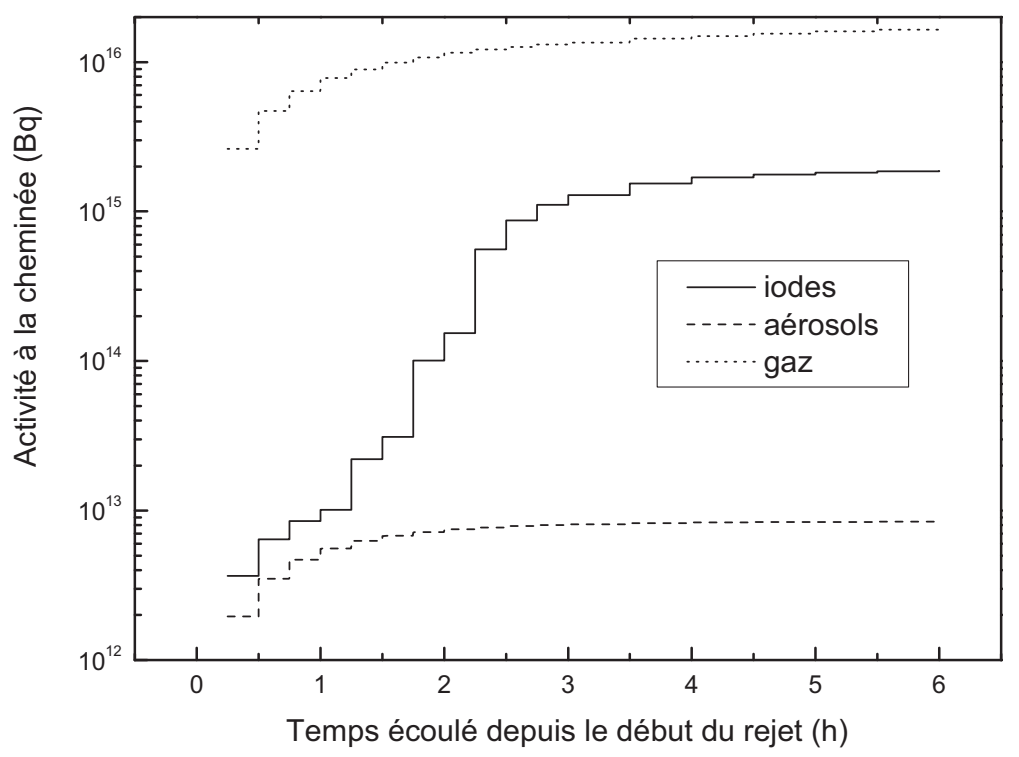

Figure 2 - Évolution des activités à la cheminée en fonction du temps depuis le début du rejet.

Evolution of activities at the outlet.

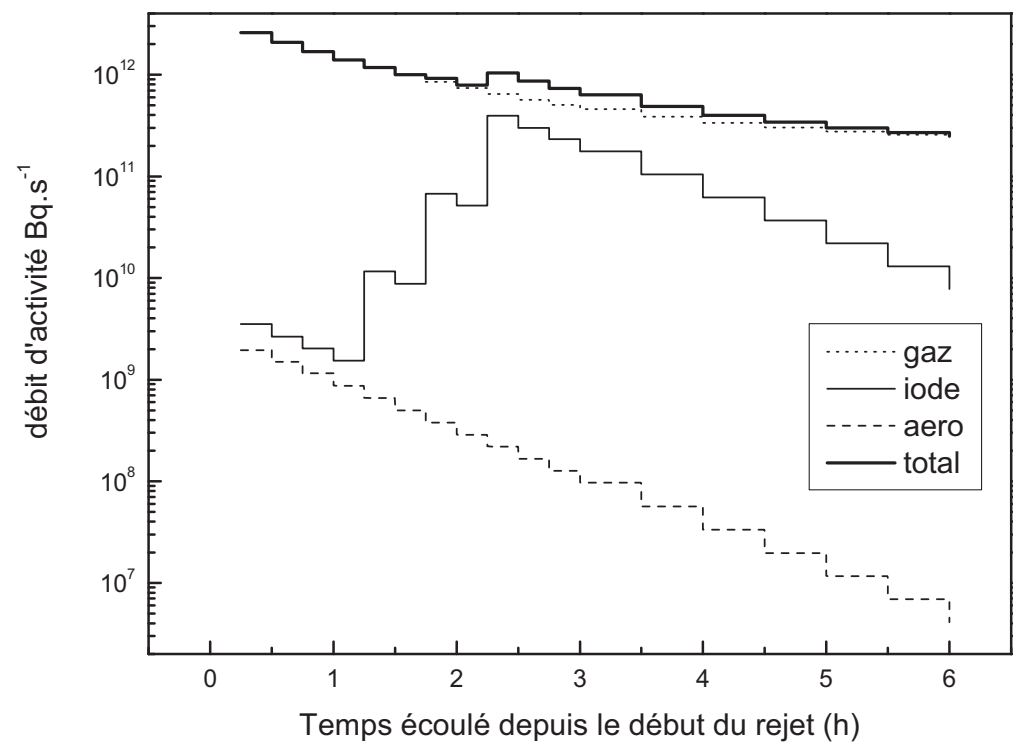

Figure 3 - Débits d'activité à la cheminée en fonction du temps depuis le début du rejet.

Evolution of activity rates at the outlet. 


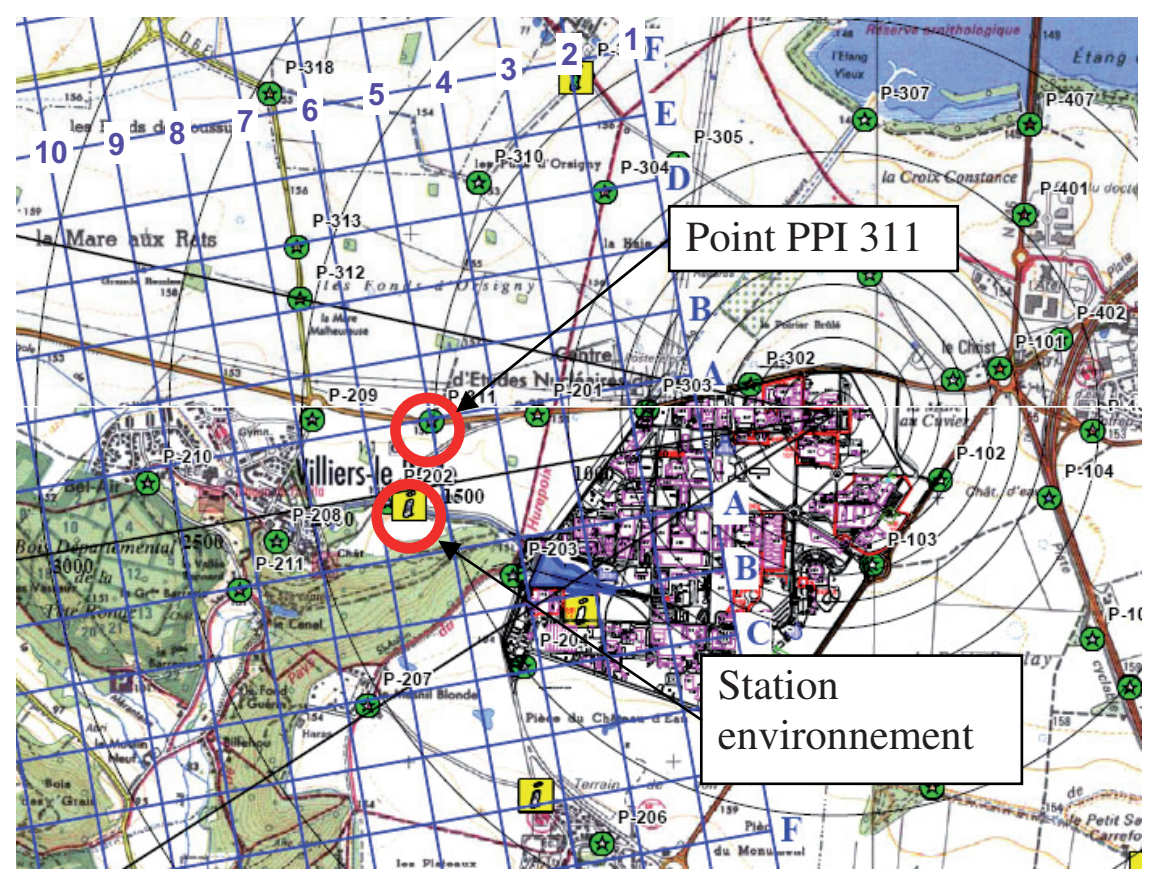

Figure 4-Maillage dans l'espace pour le calcul des CTA autour du site de Saclay.

Map for dispersion calculations around Saclay.

Pour l'exercice, ces conditions ont été fixées pendant les six heures prévues de l'exercice (vent provenant du $80^{\circ}$, diffusion normale, vitesse de vent $5 \mathrm{~m} . \mathrm{s}^{-1}$ ). Ainsi le CTA ne dépend plus du moment d'observation mais uniquement de sa position dans l'espace. Ce dernier a été maillé dans la direction du vent sur $10 \mathrm{~km}$ de long et $6 \mathrm{~km}$ de large avec un pas de 250 mètres. Chaque maille est repérée par une lettre dans l'axe du vent et un chiffre à sa perpendiculaire. Le carroyage ainsi obtenu est présenté sur la figure 4. Les CTA ont été calculés à l'aide du code CERES (Monfort, 2006) par le pôle de compétence « dispersion/impact » du CEA (DIF/DASE/SRCE) à chacune des intersections ainsi définies. 500 valeurs de CTA ont ainsi été calculées.

Sur la figure 4, sont représentés les points particuliers connus de tous les acteurs tels que les points PPI (plan particulier d'intervention) et les stations de mesure environnement du CEA/Saclay. Pour chaque point de l'espace, la valeur de CTA considérée est celle de l'intersection du maillage la plus proche. 


\subsection{Calcul des grandeurs}

Les activités, les débits d'activité pour un instant de calcul donné et l'ensemble des CTA ont été introduits dans les feuilles d'un tableur. 18 fichiers ont été établis, un par instant de calcul. Chaque fichier comporte sept onglets pour les données :

- un pour les CTA (haut de la Fig. 5) en fonction de la position exprimée dans le carroyage de la figure 4 ,

- un pour les facteurs de dose $F C D_{\text {pan,i }}$ et $F C D_{\text {depo,i }}$ (voir Éqs. (3) et (4)),

- un pour l'activité, $A_{c h}$ et le débit d'activité, $a_{c h}$ (bas gauche de la Fig. 5), de chacun des radionucléides de type gaz à la cheminée,

- un pour l'activité, $A_{c h}$, et le débit d'activité, $a_{c h}$, de chacun des radionucléides de type iode à la cheminée,

- un pour l'activité, $A_{c h}$, et le débit d'activité, $a_{c h}$, de chacun des radionucléides de type aérosol à la cheminée,

- un pour la somme de l'activité, $A_{c h}$, et du débit d'activité, $a_{c h}$, des iodes et des aérosols à la cheminée,

- un pour la somme totale de l'activité et du débit d'activité à la cheminée.

Enfin il suffit d'appliquer les formules (1) à (4) pour obtenir les grandeurs de radioprotection désirées pour les gaz, les iodes et les aérosols ou toutes combinaisons de celles-ci. La figure 5 illustre le calcul de l'activité volumique gaz au point (A,5) soit le point PPI 311 (voir Fig. 4) à $T_{0}+30$ minutes. Dans cet exemple de la figure 5 :

- les CTA en fonction de leur position dans l'espace sont représentés en haut de la figure,

- les débits d'activité et l'activité des gaz à la cheminée à $T_{0}+30$ minutes sont représentés en bas à gauche,

- le résultat, l'activité volumique gaz au point (A,5), produit du CTA et du débit d'activité des gaz est représentée en bas à droite de la figure.

\subsection{Simulation des appareils}

\subsubsection{Débit d'équivalent de dose}

La valeur du débit d'équivalent de dose est directement accessible dans l'onglet qui lui est réservé dans le tableur. En dehors de la grille présentée sur la figure 4, donc en dehors du panache, des valeurs de limite de détection, adaptées au type d'appareil, sont données aux acteurs de terrain. Pour les débits d'équivalent de dose au sol il a été considéré la somme du débit d'équivalent de dose panache (Éq. (3)) et dépôt (Éq. (4)); pour les débits d'équivalent de dose mesurés en hauteur, seul le débit d'équivalent de dose panache a été considéré. 
N. COMTE et al.

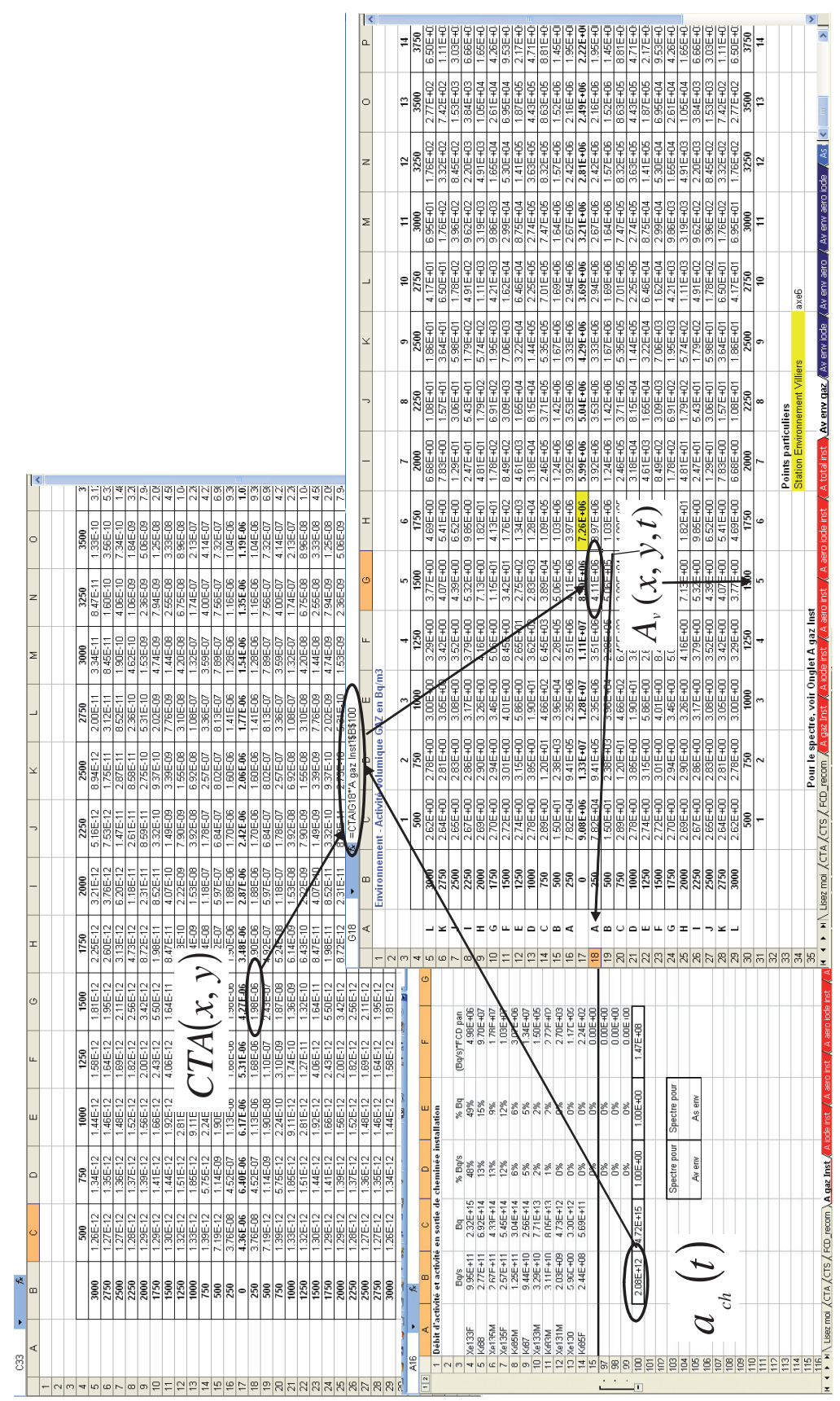




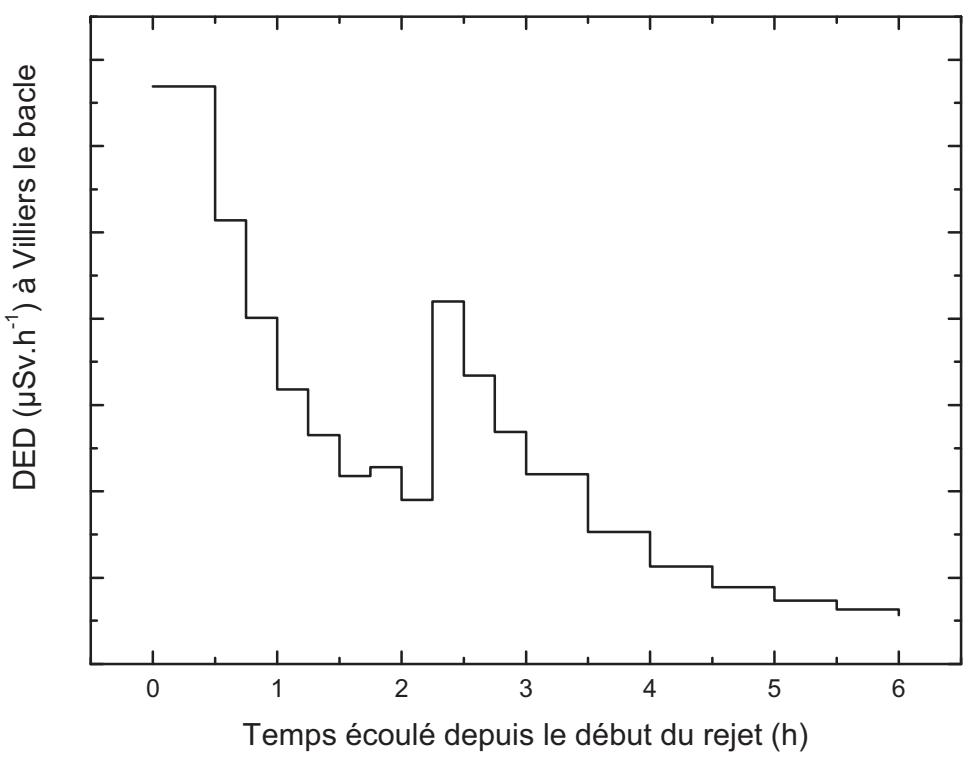

Figure 6-Simulation du débit d'équivalent de dose sur la balise de la station environnement du CEA/Saclay située à Villiers-le-Bâcle au point $(0,6)$.

Simulation of dose equivalent rate at monitoring devices at Villiers-le-Bâcle.

Certains appareils (station environnement et balise ICAM du SPR/Saclay, balises spécifiques du GIE Intra) délivrent des données en continu. Pour ces appareils, en fonction de leur localisation, un fichier informatique de type ASCII donnant le débit d'équivalent de dose en fonction du temps était délivré aux responsables des mesures. La figure 6 montre la simulation de la balise de la station environnement du SPR/Saclay située à Villiers-le-Bâcle au point $(O, 6)$ sur le carroyage de la figure 4 .

\subsubsection{Activité surfacique}

\section{Mesure directe à l'aide d'un contaminamètre}

Pour les mesures effectuées à l'aide d'un contaminamètre, une fonction de transfert a été appliquée aux valeurs calculées des activités surfaciques $\left(\mathrm{c} . \mathrm{s}^{-1} / \mathrm{Bq} \cdot \mathrm{cm}^{-2}\right)$. Ces fonctions de transferts ont été évaluées en prenant en compte le spectre isotopique du dépôt. Elles sont calculées par une méthode mise au point par le SPR/Saclay (Ratsirahonana, 1999) utilisant un code de Monte Carlo. Cette méthode fournit, pour différents appareils et différentes géométries de source, une fonction de transfert par radionucléides. 


\section{Mesure de la contamination à l'aide d'un frottis}

À l'aide d'une mesure sur frottis, il est possible de remonter au spectre isotopique (ex : spectrométrie gamma). Pour pouvoir répondre à la demande des acteurs de terrain, la première étape consiste donc à simuler le spectre gamma. La répartition du spectre ne dépend pas de l'endroit du prélèvement mais principalement du temps. Le spectre de l'activité surfacique est proportionnel à celui de l'activité intégrée calculée à la cheminée, pondéré par la vitesse de dépôt $\left(V_{d}\right)$. Ainsi, en connaissant l'activité surfacique, la surface frottée et la répartition du spectre (identique à celui de l'activité à la cheminée au même temps) il devient possible d'évaluer par proportionnalité l'activité par radionucléide sur le frottis.

\subsubsection{Activité volumique}

\section{Mesures en continu}

La valeur de l'activité volumique se lit directement sur la feuille de calcul ad-hoc du tableur.

Certains appareils (station environnement et balise ICAM du SPR/Saclay) délivrent des données en continu. Pour ces appareils, en fonction de leur localisation un fichier informatique de type ASCII donnant l'activité volumique en fonction en fonction du temps a été délivré aux responsables des mesures. La figure 7 montre la simulation de la balise de mesure de l'activité volumique des gaz de la station environnement du SPR/Saclay située à Villiers-le-Bâcle au point $(\mathrm{O}, 6)$ sur le carroyage de la figure 4.

\section{Mesures intégrées}

Certains appareils tels que les appareils de prélèvement atmosphérique (APA) ou les prélèvements d'iode et d'aérosols sur filtre fixe (PIAFF) permettent, par mesure différée de l'activité sur un filtre, de mesurer l'activité volumique intégrée. Celle-ci est donnée par l'équation (5).

Ce type d'appareil prélève entre un instant $T_{1}$ et un instant $T_{2}$. Le résultat de l'activité déposée sur le filtre, entre ces deux instants, est alors donné par l'équation (6) déduite de l'équation (5). Cette formule s'applique aux aérosols et iodes avec leurs paramètres propres (activités et rendement).

$$
A_{f}\left(x, y \text {, entre } T_{1} \text { et } T_{2}\right)=\left(A_{c h}\left(T_{2}\right)-A_{c h}\left(T_{1}\right)\right) \times d \times C T A(x, y) \times \eta
$$

Avec $\eta$ : le rendement de piégeage du filtre,

$d$ : débit de prélèvement de l'appareil de mesure. 


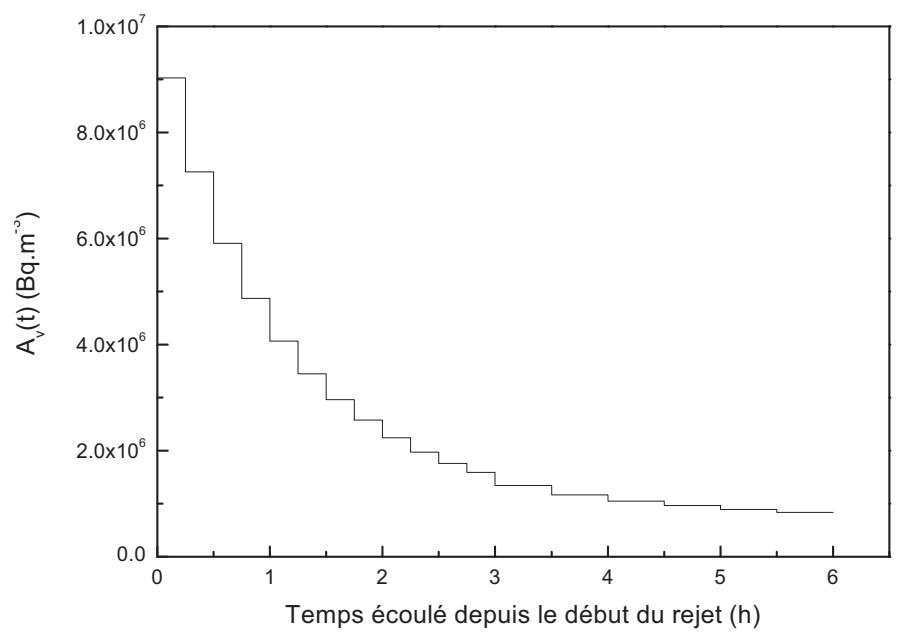

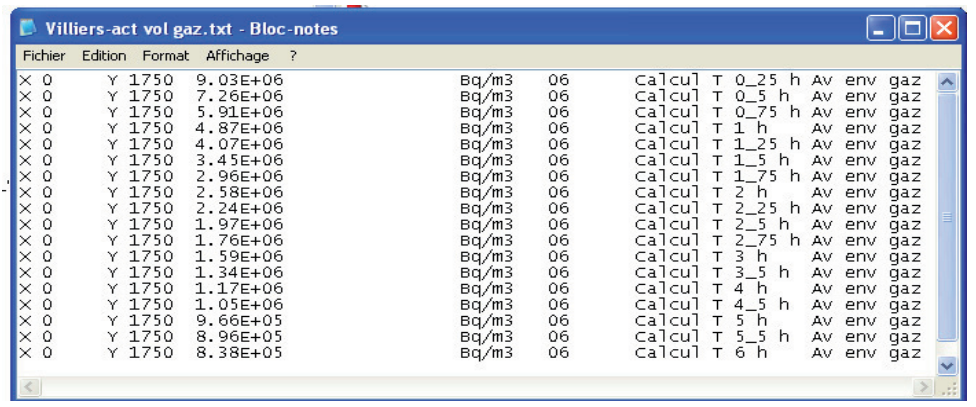

Figure 7 - Simulation de la balise de mesure de l'activité volumique des gaz de la station environnement du SPR/Saclay située à Villiers-le-Bâcle - en haut représentation graphique; en bas fichier ASCII fourni.

Simulation of gas volume activity at monitoring devices at Villiers-le-Bâcle.

Sur ce prélèvement, les acteurs de terrain peuvent effectuer une spectrométrie gamma. Il faut donc pouvoir simuler le spectre pour répondre à ce type de demande. Celui-ci ne dépend pas de l'endroit du prélèvement mais principalement du temps. Le spectre de l'activité déposée sur le filtre est proportionnel à celui de l'activité intégrée calculée à la cheminée pondérée par la vitesse de dépôt $\left(V_{d}\right)$. Ainsi, en connaissant l'activité volumique intégrée (Éq. (6)), le débit de prélèvement $(d)$, le rendement de piégeage du filtre $(\eta)$ et la répartition du spectre (proportionnel à l'activité intégrée à la cheminée) il devient possible de calculer par proportionnalité l'activité par radionucléide sur le filtre. 


\section{EXERCICE - EXERCICE - EXERCICE}

mesures au point PPI : 202

\begin{tabular}{|c|c|c|c|c|c|}
\hline & & $\begin{array}{c}\mathrm{DeD} 20 \mathrm{~cm} \\
\mu \mathrm{S} / \mathrm{h}\end{array}$ & $\begin{array}{c}\text { DeD1m } \\
\mu S v / h\end{array}$ & $\begin{array}{c}\text { DeD2m } \\
\mu S v / h\end{array}$ & $\begin{array}{l}\text { contamination surfacique } \\
\text { mesure MIP10 + sonde } \beta \\
\mathrm{C} / \mathrm{s}\end{array}$ \\
\hline $\mathrm{TO}+$ & 15 minutes & 670 & 670 & 670 & 15 \\
\hline $\mathrm{TO}+$ & 30 minutes & 510 & 510 & 510 & 26 \\
\hline $\mathrm{TO}+$ & 45 minutes & 400 & 400 & 400 & 35 \\
\hline $\mathrm{TO}+$ & 1 heure & 320 & 320 & 320 & 41 \\
\hline $\mathrm{TO}+$ & 1 heure et 15 minutes & 270 & 270 & 270 & 70 \\
\hline $\mathrm{TO}+$ & 1 heure et 30 minutes & 220 & 220 & 220 & 100 \\
\hline $\mathrm{TO}+$ & 1 heure et 45 minutes & 240 & 230 & 230 & 280 \\
\hline $\mathrm{TO}+$ & 2 heures & 200 & 190 & 190 & 420 \\
\hline $\mathrm{TO}+$ & 2 heures et 15 minutes & 460 & 420 & 420 & 1500 \\
\hline $\mathrm{TO}+$ & 2 heures et 30 minutes & 400 & 330 & 330 & 2300 \\
\hline $\mathrm{TO}+$ & 2 heures et 45 minutes & 350 & 270 & 270 & 2900 \\
\hline $\mathrm{TO}+$ & 3 heures & 310 & 220 & 220 & 3400 \\
\hline $\mathrm{TO}+$ & 3 heures et 30 minutes & 260 & 150 & 150 & 4000 \\
\hline $\mathrm{TO}+$ & 4 heures & 230 & 110 & 110 & 4400 \\
\hline $\mathrm{TO}+$ & 4 heures et 30 minutes & 210 & 90 & 90 & 4700 \\
\hline $\mathrm{TO}+$ & 5 heures & 200 & 70 & 70 & 4800 \\
\hline $\mathrm{TO}+$ & 5 heures et 30 minutes & 190 & 60 & 60 & 4900 \\
\hline $\mathrm{TO}+$ & 6 heures & 190 & 60 & 60 & 4900 \\
\hline $\begin{array}{l}\mathrm{TO}+ \\
\mathrm{DeD} 20 \mathrm{~cm} \\
\mathrm{DeD} 1 \mathrm{~m} \\
\mathrm{DeD} 2 \mathrm{~m} \\
\mathrm{C} / \mathrm{s}\end{array}$ & $\begin{array}{l}\text { le temps est donné à partir du début de } \\
\text { débit de dose mesuré à } 20 \mathrm{~cm} \text { du sol } \\
\text { débit de dose mesuré à } 1 \mathrm{~m} \text { du sol } \\
\text { débit de dose mesuré à } 2 \mathrm{~m} \text { du sol } \\
\text { contamination surfacique mesurée ave }\end{array}$ & ié à une son & & & \\
\hline
\end{tabular}

Figure 8 - Enveloppe distribuée aux acteurs de terrain donnant les simulations des mesures des appareils à différents temps de l'exercice - exemple du point PPI 202.

Example of results given to the actors for different times.

\subsection{Enveloppes distribuées aux personnels réalisant les mesures pour le compte des pouvoirs publics ou de l'exploitant}

Les personnels réalisant les mesures pour le compte des pouvoirs publics ou de l'exploitant mesurent les débits de dose (à l'aide d'un radiamètre) et la contamination surfacique (à l'aide d'une sonde de mesure $\beta$ ou X) à différents points et instants de l'incident. Aussi, pour chaque point PPI situé sur le carroyage de la figure 4, une enveloppe a été préparée et distribuée à ces acteurs, donnant les valeurs de mesures simulées pour chaque appareil à différents instants de l'exercice. Une illustration de ces enveloppes est donnée en figure 8. En dehors du carroyage, donc hors du panache, des enveloppes avec la limite de détection des appareils étaient distribuées. Les personnels réalisant les mesures avaient ainsi connaissance des niveaux de l'ambiance radiologique dans laquelle ils évoluaient, leur permettant d'adapter leur approche radioprotection lors de cet exercice. 


\section{Conclusion}

La simulation des différentes grandeurs de radioprotection pour l'exercice de crise du 17 septembre 2009 a engendré 200000 résultats de calcul et a nécessité 6 semaines de préparation. Ceci a permis aux scénaristes de répondre à l'ensemble des sollicitations des acteurs de terrain dans des délais compatibles avec les attentes. Les acteurs de terrain ont utilisé environ 250 points de mesures fictifs (dans l'espace et le temps) représentant environ 1000 grandeurs.

La distribution des enveloppes aux personnels réalisant les mesures pour le compte des pouvoirs publics ou de l'exploitant, ainsi que la simulation des mesures en continu, se sont révélées d'un grand intérêt en fournissant des valeurs cohérentes avec l'accident simulé. L'ensemble de ces mesures simulées a permis d'alimenter les différents PC opérationnels et de décision. Ainsi alimentée « en temps réel », la réflexion des différents acteurs de l'exercice n'en a été que plus riche.

La méthode présentée dans cet article est illustrée par un exemple complexe en termes de cinétique de rejet, de terme source et pour une météo constante. Elle est facilement adaptable pour tous les types de rejets; il suffit alors de calculer l'évolution de l'activité de chaque radionucléides à la cheminée en fonction du temps et de l'introduire dans les différentes feuilles d'un tableur. Cette méthode peut encore être étendue dans le cas d'une météo variable en introduisant des grilles de CTA différentes en fonction des instants de calcul.

\section{RÉFÉRENCES}

Birchall A. (1986) A microcomputer algorithm for solving compartmental models involving radionuclide transformations, Health Phys. 50 (3), 389-397.

Birchall A., James A.C. (1989) A microcomputer algorithm for solving first-order compartmental models involving recycling, Health Phys. 56 (6), 857-868.

Bourion J.P., Henry F., Lalère L., Juhasz V. (1996) Un outil de modélisation des transferts de radioactivité entre compartiment et d'évaluation des doses associées : ERASTEM. Actes du congrès SFRP «L'utilisation des codes de calcul en radioprotection », Saclay.

Comte N., Bourgois L., Bourion J.P. (2004) Organisation et schéma de calcul de conséquences radiologiques en situation accidentelle au CEA de Saclay : logiciel SENTINEL rapport interne DEN/SAC/SPR/SERD/DF/001.

Kocher D.C. (1983) Dose rate conversion factors for external exposure to photons and electrons, Health Phys. 45 (3), 665-686.

Monfort M. (2006) Présentation de la plateforme CERES, Actes du congrès SFRP «L'utilisation des codes de calcul en radioprotection », Saclay.

Monfort M., Patryl L., Armand P. (2008) Presentation of the CERES Platform used to evaluate the consequences of the emissions of radionucleides in the environment, 2008, Bergen, Norvège.

Ratsirahonana A. (1999) Méthode d'évaluation théorique des rendements de détecteurs portables munis de scintillateurs, Note interne DCEA-S/UGSP/SPR/SRI-T/99-467. 